\title{
Rafael Alberti e il pericolo di camminare per Roma
}

\author{
Elisabetta Bagli \\ Poetessa, scrittrice. Università di Roma (Sapienza)
}

https://doi.org/10.33676/EMUI_nomads.59.02

L'Andalucía, terra di contrasti, di musica e colori, di ritmi lenti e vorticosi, dotata di una natura travolgente ed esplosiva, ricca di saggezza e tradizioni, ha dato origine, nei secoli, ad artisti di differenti vocazioni, dediti alla rivelazione delle proprie esperienze di vita, del fascino indiscusso del luogo in cui nacquero, dei propri pensieri, intimi e pubblici, inerenti la società e il momento storico in cui vivevano.

Tra gli artisti, musicisti e letterati andalusi di tutte le epoche, mi preme ricordare: Manuel de Falla y Matheu, Francisco de Zurbarán, Diego de Velázquez, Bartolomé Esteban Murillo, Pablo Ruiz Picasso, Julio Romero de Torres, Federico García Lorca, Juan Ramón Jiménez, Antonio Machado, Gustavo Adolfo Bécquer, Luis de Góngora, Rafael Alberti, Vicente Alexandre, Emilio Prados, Luis Cernuda, Manuel Altolaguirre, María Zambrano e molti altri che, pur vivendo, spesso, al di fuori della propria terra natale, hanno continuato a essere fertili $\mathrm{e}$ originali creatori della cultura spagnola.

Rafael Alberti, poeta gaditano, fu uno dei membri più rappresentativi della Generazione del '27, movimento poetico che tanta rilevanza ha avuto in ambito letterario e sociale anche per le generazioni future. Alberti, come si può dedurre dal suo cognome, aveva ascendenza italiana, sebbene la sua famiglia fosse ben radicata nel Puerto de Santa María, a pochi chilometri da Cadice. La sua fu un'infanzia libera e spensierata tra le dune, le saline e il mare cristallino della sua città. Mal sopportava la scuola, perché non intravedeva nell'insegnamento altro che regole rigide che non gli permettevano di stimolare la sua più fervida fantasia. Così non finì le scuole superiori e si trasferì con la sua famiglia a Madrid seguendo la sua vocazione primordiale che era la pittura. Nella capitale spagnola 
si iniziò alla lirica poetica, proprio scrivendo dei versi innanzi al corpo esanime del padre. Da quel momento in poi, non abbandonò più quell'ispirazione che lo porterà a conoscere poeti, letterati e artisti di grande fama con i quali ebbe l'idea di rendere omaggio nel 1927, in occasione dei 300 anni della sua morte, al poeta Luis de Gongora (poeta dell'età dell'oro della Letteratura spagnola). Fu questo l'inizio della seconda "età dell'oro" - denominata "Edad de la Plata de las letras y ciencias españolas".

In seguito a uno degli episodi più sanguinosi della storia spagnola, la guerra civile (1936-1939) e all'avvento della prima e seconda Repubblica che il poeta gaditano sostenne attivamente, la poesia per Alberti divenne un modo di cambiare il mondo, un'arma necessaria per il combattimento. Alla fine della guerra, come tanti spagnoli legati a una destinazione incerta, Rafael Alberti e sua moglie, la poetessa María Teresa León, lasciarono la loro terra natale partendo per un esilio che li vide protagonisti dapprima a Parigi, poi al di là dell'Oceano Atlantico, prima in Cile, accompagnati dal loro amico Pablo Neruda, e di seguito in Argentina, a Buenos Aires, dove rimasero fino al 1962, anno in cui la situazione politica in Argentina precipitò a causa di un colpo di Stato che oltre a portare instabilità politica nel Paese non favorì la permanenza di persone coinvolte con il comunismo come il poeta e sua moglie.

Nel 1963 i coniugi Alberti si trasferirono a Roma, avvicinandosi, in questo modo, alla Spagna, tracciando un percorso immaginario verso il ritorno "a casa". Alberti decise di trasferirsi in Italia, non solo per la vicinanza alla sua terra natia e al mare tanto sospirato e amato, ma anche per la simpatia dei suoi abitanti e per l'origine dei suoi due cognomi "Alberti Merello" (i suoi nonni erano italiani, probabilmente toscani).

Lo stesso Alberti, riferendosi a quella circostanza, in un discorso di apertura di un congresso presso la storica Universidad de Alcalá de Henares, disse: "Il 28 maggio 1963, dopo quasi 24 anni di esilio nella Repubblica Argentina, stavo entrando attraverso l'immensa Porta del Paradiso, nella città di Roma. All'epoca avevo 61 anni. E alcune ansie e desideri angoscianti di immergermi, di perdermi, di restringermi fino a scomparire in quel complicato e pericoloso labirinto di piazzette e vicoli del quartiere che scelsi come alloggio, il romanesco Trastevere, allegra capitale, dentro Roma, dei gatti, dei ratti, dei rumori rapidi, delle urla nei 
bar nei pomeriggi di calcio e, tra le altre cose attraenti e insospettate, delle catene montuose di non profumate pile di immondizia, ammucchiate agli angoli delle strade. Entrai a Roma attraverso la porta del cielo, come fece, circa quattro secoli prima di me, nel 1559 all'età di 22 anni, Miguel de Cervantes attraverso la Porta del Popolo, baciando una e mille volte le soglie e i margini dell'ingresso, salutando la città con le lacrime agli occhi"1.

La famiglia Alberti stanziò la sua sede familiare net quartiere di Trastevere dove rimase fino al 1977, due anni dopo il termine della dittatura del generale Francisco Franco, segnando il suo ritorno in Patria.

Una volta in Spagna, Rafael Alberti si rese conto di quel che stava succedendo intorno alla sua figura e alla sua arte: molti si ricordavano di lui, non solo i suoi vecchi amici, ma anche le nuove generazioni leggevano le sue opere con grande interesse. Nella sua terra natale partecipò a varie conferenze e tributi alla sua persona e ai poeti del '27, ricevendo premi di ogni genere e rifiutando, per coerenza e convinzione repubblicana, il Premio Principe de Asturias. Morì nel 1999 nella sua casa de El Puerto de Santa María, vicino alle spiagge e al mare della sua infanzia, disponendo che le sue ceneri venissero sparse nelle acque che gli appartenevano.

Rafael Alberti fu un artista completo, amante della musica, del teatro e della pittura, la sua prima vocazione artistica. Pubblicò moltissime opere poetiche e teatrali delle quali alcune illustrate da lui stesso. Di grande rilievo possono considerarsi: "Marinero en tierra”, "Cal y canto", "La arboleda perdida. Memorias",

\footnotetext{
1 "El día 28 de mayo de 1963, después de casi 24 años de exilio en la República Argentina, hacía mi entrada a través de la inmensa Puerta del Cielo, en la ciudad de Roma. Yo tenía entonces 61 años. $Y$ unas ansias, unos deseos angustiosos de sumergirme, de perderme, de estrecharme, hasta desaparecer en aquel complicado y peligroso laberinto de plazuelas y callejones del barrio que elegí como vivienda, el romanesco Trastevere alegre capital, dentro de Roma, de los gatos, las ratas, los veloces ruidos, el griterío de los bares en las tardes de futbol y, entre muchas otras cosas atrayentes e insospechadas, las cordilleras de los no muy perfumados montones de basuras, hacinados en las esquinas. Yo entré en Roma por la puerta del cielo, como cuatro siglos antes, en 1559, a la edad de 22 años, entró Miguel de Cervantes por la Puerta del popolo, besando primero una y muchas veces, los umbrales y márgenes de la entrada, saludando a la ciudad con lágrimas en los ojos ("Rafael Alberti [1902-1999]")." Traduzione italiana a cura di Elisabetta Bagli. II Congreso Internacional de Literatura y Cultura Españolas Contemporáneas "Roma, peligro para caminantes. La representación de una ciudad en estratos" - Ester Hernández Palacios Mirón Universidad Veracruzana, pagina 1.
} 
"A la pintura: Poema del color y la línea", "Sobre los ángeles", "El Adefesio", "Roma, peligro para caminantes", "Canciones de alto Valle del Aniene".

Alberti, come capita sempre nell'evoluzione artistica, attraversò varie tappe nella sua poetica: dapprima vi fu quella classica e tradizionale; poi la surrealista, pienamente compenetrato in un linguaggio simbolico e onirico; di seguito vi fu una fase in cui la poesia non poteva essere concepita senza una ragione politica e rivoluzionaria; poi vi fu la fase ironica, fino ad arrivare a quella in cui la nostalgia per la sua Patria risúltava costituire l'asse portante di ogni suo verso: la poesia dell'esilio.

A Roma, Rafael Alberti sentiva una grande nostalgia per la sua terra natale, ma anche per quella che lo aveva adottato, l'Argentina. In lui, nel suo intimo, si mescolavano il desiderio di essere altrove e la necessità e l'impazienza di conoscere la vera essenza della città "eterna", osannata dal mondo intero per la sua ricchezza artistica e monumentale, insultata e assaltata dai suoi abitanti nel labirintico fervore della vita quotidiana.

La silloge poetica "Roma, peligro para caminantes" ("Roma, pericolo per i viandanti", così tradotta da Vittorio Bodini, uno dei traduttori italiani ufficiali di Alberti) venne pubblicata per la prima volta nel 1968 in Messico; nel 1976 venne pubblicata in versione ampliata, e nel 1977 venne effettuata la sua ultima ristampa. II titolo, molto curioso, lo si deve al fatto che il poeta diceva di "correre un serio pericolo" ogniqualvolta si trovava ad attraversare i vicoli di Roma. II "pericolo", come possiamo supporre, altro non era che una metafora della quotidianità in cui il poeta era immerso, una vita completamente diversa da quella di tanti artisti che avevano deciso, in varie epoche, di installarsi nella capitale italiana. La sua non era la Roma di Goethe o di Stendhal, bensí era una Roma bohémien e proletaria, di mercatini e di graffiti sui muri. La Roma di Alberti era quella in cui il suono delle fontane si mescolava alle grida esilaranti degli abitanti, quella Roma sparita degli acquarelli di Ettore Roesler Franz, le cui pennellate vigorose e pungenti del poeta riuscivano a graffiare con forza la genuinità del paesaggio descritto dai suoi versi vivaci e incisivi, regalando al lettore quelle emozioni contrastanti necessarie a comprendere la complessità della sua anima indomita e anticonformista, amplificata dalla città eterna. 
Di quest'opera dedicata a Trastevere, Alberti disse: "Posso confessare che nel mio amato quartiere dovetti diventare un torero, allenandomi ad assottigliarmi, a dimagrire contro i muri, a uscire in piedi, a correre veloce come se fossi davanti a un toro, mentre vedevo arrivare quelle esalazioni interplanetarie, cieche e senza preavviso, attraverso strade così strette e vicoli così tortuosi. Per questo, a poco più di un anno di coraggiosa vita romana, è nato un libro, intitolato con precisione astronomica: "Roma, pericolo per i viandanti" (titolo preso dal libro tradotto da Vittorio Bodini ${ }^{2}$ ). Ora spero che un giorno, in qualche festa o anniversario, il comune della Città Eterna incida, in un vicolo non lontano dalla mia via Garibaldi, una targa che dice: «Vicolo di Rafael Alberti (prima del Cinque, del Cedro, ecc.)», visto che mi sono stabilito qui, sono diventato un vicino di casa di questo quartiere per cantarlo umilmente e simpaticamente, allontanandomi dalla Roma monumentale, amando solo quella non ufficiale, la più "antigoethiana" che si possa immaginare: la Roma trasteverina degli artigiani, delle pareti rotte, dipinte di iscrizioni politiche o d'amore, la Roma segreta, statica, notturna e, repentinamente, muta e solitaria ${ }^{3 "}$.

L'esilio produsse una lesione interiore talmente forte da portare il poeta verso una constante ricerca della sua identità, portandolo a vagare come un cieco senza meta (immagine ricorrente nel libro di Alberti), intensificando quel senso di vuoto a cui vuole sopperire camminando per le strade di Roma e osservando quel che lo circonda per entrare in contatto, quasi fisico, con quei luoghi che lo avrebbero aiutato a ritrovarsi attraverso la creazione artistica.

\footnotetext{
2 Vittorio Bodini, poeta e traduttore ufficiale, nonché amico di Rafael Alberti.

3 "Puedo confesar que en mi amado barrio tuve que volverme torero, adiestrándome en ceñirme, en adelgazarme contra los muros, en salir por pies, corriendo veloz como ante un toro, al ver llegar aquellas exhalaciones interplanetarias, ciegas y sin aviso, por tan estrechas calles $y$ retorcidos callejones. De ahí nació, a poco más de un año de vida romanesca valerosa, un libro, titulado con astronómica exactitud: Roma, peligro para caminantes. Ahora espero que algún día, en alguna fecha de aniversario, el commune de la Ciudad Eterna estampe en algún vicolo, no lejano de mi Vía Garibaldi, una placa que diga: «Vicolo di Rafael Alberti (antes del Cinque, del Cedro, etcétera)", porque yo me instalé aquí, me convertí en vecino de este barrio para cantarlo humildemente, graciosamente, rehuyendo la Roma monumental, amando sólo la antioficial, la más antigoethiana que pueda imaginarse: la Roma trasteverina de los artesanos, los muros rotos, pintarrajeados de inscripciones políticas o amorosas, la secreta, estática, nocturna y, de improviso, muda y solitaria." Traduzione italiana a cura di Elisabetta Bagli. El País- TRIBUNA: "LA ARBOLEDA PERDIDA" - De Buenos Aires al Trastevere 11 de noviembre de 1984, autor Rafael Alberti.
} 
Vagare per la città senza meta, ma con il solo scopo di conoscere il contesto socio-culturale in cui era immerso, era fondamentale per Alberti, perché oltre a essere un atto di solitudine volto alla riflessione intima su una realtà tutta da interiorizzare, era soprattutto un atto di libertà che lo allontanava dalla frenesia di un luogo che gli incuteva paura, rispetto, ma anche ironia e tanta voglia di vivere ${ }^{4}$.

Le poesie che compongono questa silloge ${ }^{5}$, come avviene nelle liriche incluse in altri libri della vasta produzione di Rafael Alberti, hanno una spiccata connotazione neo-barocca 6 . La silloge "Roma, peligros para caminantes" è divisa in quattro sezioni: "X sonetti", "Versi sciolti, scene e canzoni", "X sonetti" e "Poemas con nombres".

L'introduzione è affidata a una poesia intitolata “Monserrato 20", l'indirizzo della prima casa del poeta nello straordinario quartiere Trastevere. Analizzando questa poesia possiamo vedere che il poeta scende dalle scale che separano la sua casa dalla città di Roma per entrare non solo nelle strade della città eterna e curiosare, ma con la volontà di instaurare un vero e proprio dialogo con lei. Si può dire che il poeta, nel suo immaginario, sentiva Roma come un essere vivo (poteva essere un gatto, considerando che le strade della città eterna, da sempre, sono abitate da tanti piccoli felini randagi e la stessa copertina dell'edizione del libro alla quale si fa riferimento in questo scritto, copertina di Alberto Corazón, raffigura un gatto) al quale parlare, con il quale ridere e arrabbiarsi, con il quale diventare amico e confidente, raccogliendo le sue indiscrezioni, interiorizzandole ed esternandole in versi, spesso beffardi e irriverenti. Essendo Roma

\footnotetext{
${ }^{4}$ Pag 107

${ }^{5}$ In questo breve saggio si fa riferimento a "Roma, peligro para caminantes", Rafael Alberti, Ed. Seix Barral, ed. 1968, prima edizione nella Biblioteca Breve de Bolsillo: aprile 1976. $1^{\text {a }}$ ristampa: maggio 1977.

6 "II Neobarocco - scrive il professor Omar Calabrese, famoso semiologo e critico d'arte italiano, riconosciuto internazionalmente- non è un movimento artistico o una tendenza, ma è lo spirito stesso dell'epoca che viviamo, è un fenomeno culturale, un atteggiamento, uno spirito al quale, com'è ovvio, non solo la letteratura, i mass-media, il cinema, ma anche le arti visive si adeguano, anzi ne occupano un ruolo particolarmente significativo". Calabrese individua i principi del Neobarocco nel Ritmo e nella Ripetizione - si pensi all'estetica della ripetizione, appunto, fondata sugli elementi della variazione organizzata, dell'irregolarità regolata, del ritmo velocissimo - 0 nell'Eccesso - quella tensione, questa volta centrifuga, al limite: quella messa in discussione di una qualche regola che tende a destabilizzare il sistema -, nel Pressappoco e Non so che - il fascino dell'approssimazione, quanto nelle scienze tanto nelle filosofie -, nel Nodo e Labirinto, nella Distorsione e Perversione, nella cura del Dettaglio e del Frammento. Per Calabrese non vi sarà più il caos come viene spiegato nella mitologia greca antica quale origine dell'Universo, ma un totale cambiamento di gusto per cui i fenomeni caotici vengono considerati esteticamente belli.
} 
rappresentata come una persona (o un gatto), Alberti le da la stessa capacità di ascolto che hanno le persone e per questo le parla o, addirittura, interagisce con lei. Dalla lettura delle poesie contenute in questa silloge si può notare come Alberti e Roma siano esseri dotati di una forte alchimia, le cui azioni costituiscono il detonante per intraprendere quel percorso che sfocerà nelle composizioni in versi?

Attraverso le caratteristiche elencazioni di oggetti eterogenei, tipiche del neobarocco, il poeta si avvia a districare delle matasse con l'uso della parola scritta e lo fa come se dovesse riavvivare le setole secche di pennelli, intingendole nell'acqua per poi strofinarle di nuovo su quei colori da lui descritti prima di dare le sue pennellate caratteristiche, quasi a suggellare l'intima connessione della poesia con l'arte pittorica ${ }^{8}$. "La pittura è una poesia silenziosa e la poesia è un dipinto che parla", ci disse il poeta greco Simonide di $\operatorname{Ceos}^{9}$ : l'arte di Rafael Alberti ne incarna pienamente il significato.

In "Roma, pericolo per i viandanti", il poeta andaluso usa le parole quasi fossero delle pennellate, fluide, arricchite da violenti contrasti, come se delle rette parallele si alternassero a rette incidenti, come se dietro alle curve sfociasse sempre una nuova Roma, insolita e originale, dal carattere conciliante e superficiale, ma nel contempo pungente e aggressivo, come se quei colori e quelle luci producessero i suoni delle parole facendo sgorgare dalle stesse quelle tonalità cromatiche indispensabili alla descrizione dell'effervescente e animata atmosfera di un quartiere popolare come Trastevere.

Ed è proprio al trasteverino Giuseppe Giocachino Belli (1791-1863), poeta irriverente e sarcastico, grande icona, che Rafael Alberti dedica la prima sezione

\footnotetext{
7 Vid. II Congreso Internacional de Literatura y Cultura Españolas Contemporáneas "Roma, peligro para caminantes. La representación de una ciudad en estratos" - Ester Hernández Palacios Mirón Universidad Veracruzana, pagina 2.

8 "Rafael Alberti y la poesía tradicional" - Armando López Castro- Universidad de León pag. 107.

9 Simonide di Ceos è stato il primo a stabilire il confronto tra pittura e poesia all'incirca nell'anno 470 prima della nostra era. Questo è ciò che abbiamo appreso da Plutarco quando ci dice che il poeta ha stabilito un simile confronto perché a suo avviso: "le azioni che i pittori rappresentano mentre accadono, le parole le presentano e le descrivono quando sono già avvenute". Quindi, mentre il pittore deve presentare il suo modello nel momento in cui realizza un dipinto, basterebbe solo che il poeta sia presente al momento per poi ricostruirlo e renderlo immortale attraverso la sua memoria e la tecnica delle sue parole. Per questo Simonide è anche riconosciuto come uno dei genitori della mnemonica.
} 
del libro summenzionato, iniziando con lo stesso poeta romano un dialogo in forma di sonetti, la forma poetica tanto amata dal Belli. Da sfondo a questo dialogo vi è lo splendore antico di Roma, i suoi elementi popolari frammisti all'imponenza di una città che è essa stessa Storia.

La Roma che si intuisce nelle poesie di Alberti è, senza ombra di dubbio, di stampo felliniano, quella della "Dolce Vita" o di "Otto e mezzo", per intenderci, quella in cui le meravigliose ed eleganti piazze convivono coi i rumori tipici delle macchine e delle motociclette, in cui i personaggi storici e famosi che hanno reso importante la città convivono con i ladri, i ratti, i gatti e le "gattare" romane. Per Alberti, Roma rappresenta un labirinto nel quale muoversi e perdersi a ogni ora, nel quale "ampliarsi" e "stringersi" a seconda dell'ampiezza delle strade percorse, diluendosi nel caos dei suoi frammenti ${ }^{10}$ :

"Monserrato 20"1

Oh, Roma desiderata, mi tieni in te,

già sono dentro di te e tu dentro di me ti trovi!

Mi ingrandisco o mi assottiglio per le strade e per le piazze

di questo quartiere in cui vivo vicino al fiume,

Già sono dentro di te, a tutte le ore

in te mi muovo, nuova lingua tua,

Roma nella notte, oscura voce di fontana,

\footnotetext{
10 Vid. II Congreso Internacional de Literatura y Cultura Españolas Contemporáneas "Roma, peligro para caminantes. La representación de una ciudad en estratos" - Ester Hernández Palacios Mirón Universidad Veracruzana, pag. 3

11 "¡Oh, Roma deseada, en ti me tienes, /ya estoy dentro de ti, ya en mí te encuentras!/ Me agrando o adelgazo por las calles y plazas/de este barrio que habito junto al río,... Ma estoy dentro de ti, ya a todas horas/en ti me muevo, nueva lengua tuya,/Roma en la noche, oscura voz de fuente,/Roma en la luz, clara canción del día./ Quiero perderme en medio de tu aliento,/ ser aire popular entre tus aires" (Monserrato 20, "Roma, peligro para caminantes" pag.7). Traduzione italiana a cura di Elisabetta Bagli.
} 
Roma con la luce, chiara canzone del giorno.

Voglio perdermi in mezzo al tuo respiro,

essere aria popolare tra le tue arie.

Nelle poesie di Alberti vi è grande ironia, nonché un interesse specifico alla sperimentazione delle parole da lui adoperate con insolente vitalità, la stessa di una Roma rozza e grossolana che, nonostante tutto, risorge sempre alla sua funzione primordiale di musa ispiratrice per artisti e letterati, attraverso il marmo e i monumenti che la videro brillare nel Rinascimento e che, ancora, ne costituiscono la sua ossatura e il suo incanto.

Nel dialogo con il Belli, Alberti dice di essere "figlio dei mari di Cadice, nipote di Lope, Góngora e Quevedo" e chiede il permesso di mettere nelle mani del "Padrone di casa" (lo stesso Belli) la sua opera:

"Monserrato 20"12

Lascia, Belli, amico mio, che nelle tue mani

metta, ora che ha già perso la paura,

i suoi sonetti romani

un figlio dei mari di Cadice,

nipote di Lope, Góngora e Quevedo

I dieci sonetti della prima sezione si aprono tutti con dei brevi versi del Belli espressi nella sua lingua originale, il romanesco, a suggellare lo stretto rapporto tra i poeti e tra loro e Roma. I testi del Belli aprono i sonetti a lui dedicati quasi a preannunciarne il contenuto.

12 "Deja, mi Belli amigo, que en tus manos/te ponga ahora, ya perdido el miedo,/sus sonetos romanos/un hijo de los mares gaditanos,/nieto de Lope, Góngora y Quevedo" (Monserrato 20, op. cit. pag. 9). Traduzione italiana a cura di Elisabetta Bagli. 
Alberti entra in questo "essere"(Roma), vagando attraverso i suoi innumerevoli volti, osservandoli nella loro quotidianità, cantandoli con ironia e descrivendoli, spesso, in modo irriverente:

“Campo de' fiori” 13

Campo de' Fiori, campo di fiori,

rivenditore di tutti i colori,

adulatore di grazie, luce, trambusto...

Come il più triste re dei mercati,

sui tuoi fuochi vivi, già spenti,

ancora arde Giordano Bruno.

"Vita poetica" 14

Leggi il giornale e soffri perché tutto

se non è carta igienica è latrina,

vomito incenso, crimine e salvietta.

Piangi che non sia andata in altro modo

ciò che servì a ingozzarti e a ingrassare...

13 "Campo de' Fiori, campo e las flores,/repartidor de todos los colores, gracias requiebro, luz, algarabía...//Como el más triste rey de los mercados,/ sobre tus vivos fuegos, ya apagados,/arde Giordano Bruno todavía". (Campo de'Fiori, op. cit. pag. 16). Traduzione italiana a cura di Elisabetta Bagli

14 "Leer el diario y lamentar que todo/si no es papel higiénico es retrete,/crimen, vómito, incienso, servilleta.//Llorar porque no ha sido de otro modo/lo que ya se fue en panza y en el moflete.../ésta en Roma es la vida de un poeta." (Vida poética, op. cit. pag.17). Traduzione italiana a cura di Vittorio Bodini. 
questa è in Roma la vita di un poeta.

"Arte sacra romana". 15

Dio, fa' che Michelangelo, improvvisamente,

si svegli e, tornando dalla morte,

con un feroce martello in mano, una mattina,

a colpi e senza pietà, ti faccia in mille pezzi,

per sollevarti di nuovo a martellate,

Cristo virile, tra il gregge romano.

Nella stesura di questi dieci sonetti iniziali Alberti persegue senz'altro l'obiettivo di proteggere lo straniero, nel suo vagare per le strade di Roma, difendendolo dall'essere divorato dalla "mostruosa" città piena di storia, di arte e di monumenti, ma anche di sudiciume e invereconda umanità:

"Roma, pericolo per i viandanti 16 "

Cerca di non guardare i suoi monumenti,

viandante, se verso Roma t'incammini,

apri cent'occhi, le pupille affina,

15 "Haz, Dios, que Miguel Ángel se despierte,/de súbito y, volviendo de la muerte,/ feroz martillo en mano una mañana,//a golpes sin piedad te haga pedazos,/para alzarte de nuevo a martillazos,/Cristo viril, entre la grey romana" (Arte sacra romana, op. cit. pag. 18). Traduzione italiana a cura di Elisabetta Bagli.

16 "Trata de no mirar sus monumentos,/caminante, si a Roma te encaminas/ Abre cien ojos, clavas cien retinas,/esclavo siempre de los pavimentos./ Trata de no mirar tantos portentos, fuentes, palacios, cúpulas, ruinas, pues hallarás mil muertes repentinas..." (Roma, peligro para caminantes, op. cit. pag. 14). Traduzione italiana a cura di Elisabetta Bagli. 
schiavo soltanto dei suoi pavimenti.

Cerca di non guardare così tante meraviglie,

fontane, palazzi, cupole, rovine,

perché incontrerai mille morti repentine...

"Finalmente"17"

Tutto ti infilza, tutto ti intrappola,

giuri per Bacco, il Papa, la Madonna...

E a Roma, infine, fai la dolce vita.

I riferimenti alla sua Andalucía e ai suoi trascorsi in Argentina non mancano nella prima sezione e in tutto il libro. Nella poesia "Ciò che ho lasciato per te (Lo que dejé por ti)" il dolore per la lontananza dalle due terre, che con i loro elementi hanno costituito il fulcro identitario dell'anima di Alberti, si unisce alla necessità, mai domata, di incontrare un luogo di appartenenza. Alberti lo fa in questa lirica ricorrendo all'anafora "dejé” (lasciai), alle immagini pittoriche innate nel suo modo di scrivere, personificando, in modo assolutamente diretto, la città di Roma ${ }^{18}$ :

"Ciò che ho lasciato per te" 19

\footnotetext{
17 "Todo te ensarta, todo te empitona, /juras por baco, el papa, la Madona.../ Y en Roma al fin haces la dolce vita." (Al fin, op. cit. pag.20). Traduzione italiana a cura di Elisabetta Bagli.

18 Vid. «Già troppe volte esuli», Letteratura di frontera e di esilio, a cura di Novella di Nunzio e Francesco Ragni Tomo I copyright (c) Università degli Studi di Perugia, AA.VV. Forme di esilio, migrazione, frontera: poesia, Abele Longo. Roma, viandanza dellesilio. Rafael Alberti tradotto da Vittorio Bodini, pagg. 112-113

19 "Dejé por ti mis bosques, mi perdida/arboleda, mis perros desvelados,/mis capitales años desterrados/hasta casi el invierno de la vida.//Dejé un temblor, dejé una sacudida,/un resplandor de fuegos no apagados,/dejé mi sombra en los desesperados/ojos sangrantes de la despedida.//Dejé palomas tristes junto a un río,/caballos sobre el sol de las arenas,/dejé de oler la mar, dejé de verte.//Dejé por ti todo lo que era mío./Dame tú, Roma, a cambio de mis
} 
Lasciai per te i miei boschi, la tradita

fila d'alberi, i cani vigilanti,

e gli anni dell'esilio più importanti

fino a quasi l'inverno della vita.

Lasciai un sussulto, lasciai un tremolio,

un fulgore di fuochi non smorzati,

e l'ombra mia lasciai nei disperati

occhi che sanguinavano all'addio.

Lasciai colombe tristi accanto al rio,

cavalli sotto il sole delle arene,

senza odore del mar, senza vederti.

Lasciai per te tutto ciò che era mio.

Dammi tu, Roma, in cambio delle pene

tutto ciò che ho lasciato per averti.

"Versi sciolti, scene e canzoni" è il titolo della seconda sezione del libro. I versi sciolti sono poesie divise in frammenti slegati tra loro, ognuno dei quali è contrassegnato da un numero consecutivo, sono come delle vignette nelle quali

penas,/tanto como dejé para tenerte." (Lo que dejé por ti, op. cit. pag. 13). Traduzione italiana a cura di Vittorio Bodini. 
viene dipinta una scena o descrivono, con una sola pennellata, un personaggio, vero o immaginario, un'emozione ${ }^{20}$ :

Oh Roma dalle porte giganti degli dei!

Oggi ho visto Polifemo uscire da una di esse. ${ }^{21}$

Sebbene l'umorismo la faccia da padrone, in questi versetti della seconda sezione si rivela un tono maggiormente nostalgico e melanconico.:

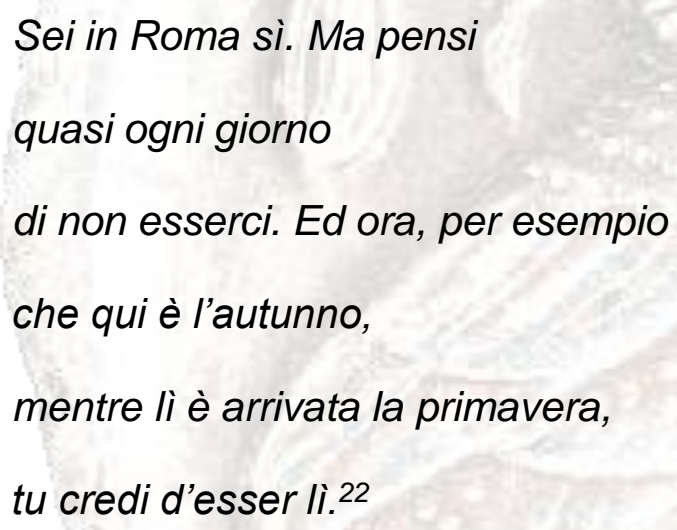

In questi frammenti lirici risiede un aspetto per certi versi commovente: Alberti era consapevole di dover ricominciare tutto d'accapo, di dover definire di nuovo se stesso in un luogo diverso che non era né la sua Patria né l'Argentina che per

20 Vid. II Congreso Internacional de Literatura y Cultura Españolas Contemporáneas "Roma, peligro para caminantes. La representación de una ciudad en estratos” - Ester Hernández Palacios Mirón Universidad Veracruzana, pag. 3

214 iOh Roma de las puertas gigantes para dioses!/Hoy vi salir por una a Polifemo, op. cit. pag. 26. Traduzione italiana a cura di Elisabetta Bagli.

227 "Tú estás en Roma, sí. Pero tú piensas,/casi todos los días,/que no lo estás. Ahora, por ejemplo,/que es el otoño aquí,/aunque allí ya llegó la primavera,/piensas que estás allí." , op. cit. pag. 48. Traduzione italiana a cura di Vittorio Bodini. 
tanti anni lo aveva accolto come un figlio, ma era, comunque, un'altra terra straniera ${ }^{23}$.

Le "scene" (sottotitolo poesie sceniche) dipingono dei "quadri" in cui si intrecciano la Roma storica e monumentale con quella contemporanea, nella quale i personaggi della città dialogano tra loro e partecipano attivamente ai vari episodi quotidiani, siano essi persone, monumenti o animali (come accade in "I due amici (Los dos amigos)", tra il cocchiere e il suo cavallo, ad esempio) ${ }^{24}$ :

"I due amici" 25

Santa María in Trastevere... ti piace

questa fontana, lo so... con quanto gusto

berresti ora in uno dei suoi getti ...

Come mangia la gente! Quante macchine!

Sono i nostri nemici...

Dai, su, Giorgio! Non addormentarti, bambino...

Siamo già arrivati.

Sono triste, sai?

Ma solo a te, Giorgio, mio cavallo,

posso dirlo senza vergognarmi.

\footnotetext{
${ }^{23}$ Vid. «Già troppe volte esuli», Letteratura di frontera e di esilio, a cura di Novella di Nunzio e Francesco Ragni Tomo I copyright (C) Università degli Studi di Perugia, AA.VV. Forme di esilio, migrazione, frontera: poesia, Abele Longo. Roma, viandanza dellesilio. Rafael Alberti tradotto da Vittorio Bodini, pagg. 112-113

24 Vid. II Congreso Internacional de Literatura y Cultura Españolas Contemporáneas "Roma, peligro para caminantes. La representación de una ciudad en estratos" - Ester Hernández Palacios Mirón Universidad Veracruzana, pag. 4

25 "Santa María in Trastevere... Te gusta /esta fuente, lo sé... Con cuánto gusto/ beberías ahora en uno de sus chorros.../ ¡Cómo come la gente! ¡Cuántos autos!/ Son nuestros enemigos... /¡Vamos, arre, Giorgio! No te duermas, niño... /Hemos llegado ya. /Tengo tristeza, ¿sabes?/ Mas sólo a ti, Giorgio, mi caballo,/ te lo puedo decir sin que me dé vergüenza" (Los dos amigos, op. cit. pag. 63). Traduzione italiana a cura di Elisabetta Bagli.
} 
Un altro poema scenico ricrea il mondo di Francesco Delicado sia dal punto di vista ambientale sia per quel che concerne i personaggi. Alberti riconosce in una donna che cammina per la Piazza di Campo de' Fiori la Lozana andaluza protagonista del romanzo picaresco di Delicado26. Con la sua lirica "La puttana andaluza"27, il poeta ha mantenuto lo spirito popolare del romanzo di Delicado attraverso l'uso di un linguaggio ironico ed esacerbato che riflette la vita quotidiana dei quartieri che frequenta la lozana, dando luogo a delle istantanee in cui vengono rappresentati i costumi e le abitudini di Roma e dei suoi abitanti. I tempi in cúi si muovono i due autori sono diversi, ma l'anticlericalità è tangibile in entrambe le opere e diventa persino più convincente nella rielaborazione teatrale della stessa opera scritta da Alberti28.

In “Sarà un crimine (¿Será un crimen?), a mio modo di vedere, una delle più belle liriche di questo libro, il poeta andaluso parla della sua vita romana e dell'amore per questa città nella quale si confonde tra le fontane e gli alberi, trasformandosi egli stesso nel rumore dell'acqua che sgorga o nella bellezza dei fiori che colorano la città e lo fa chiedendosi se è un crimine voler darsi un respiro quotidiano per rimanere in contemplazione delle bellezze di Roma ${ }^{29}$ :

\section{"Sarà un crimine?"30}

26 Francisco Delgado che cambiò inl suo nome in Delicato (c. 1475-c. 1535) fu un sacerdote, medico, editore e scrittore spagnolo dell'epoca Rinascimentale. Visse tra le altre città a Roma e a Venezia. La sua opera più famosa fu el Retrato de la Lozana andaluza publicato nel 1528.; si tratta di un romanzo picaresco dai toni maliziosi ed esuberanti in forma dialogata. L'obiettivo principale di quest'opera era quello di criticare i vizi della Roma rinascimentale, più concretamente del Papa Clemente VII dei Medici. II suo linguaggio è pieno di italianismi, di dilaetto romano e anche di dialetto andaluso della provincia di Jaén.

27 Op. cit. pag. 29

28 Vid. «Già troppe volte esuli» Letteratura di frontera e di esilio, a cura di Novella di Nunzio e Francesco Ragni Tomo I copyright (c) Università degli Studi di Perugia, AA.VV. Forme di esilio, migrazionem frontera: poesia, Abele Longo Roma, viandanza dell'esilio, Rafael Alberti tradotto da Vittorio Bodini, pag. 112-113

29 "Rafael Alberti, caminante en Roma”, Marta Villarino, pag 258/ CELEHIS

30 "Será un crimen sentarse en la mañana/ a escuchar la palabra de las fuentes,/llegar a ser rumor, a ser el eco/de un susurro sin fin ensimismado?// ¿Un crimen resbalar sobre los árboles/los ojos, descenderlos de las copas,/volcarlos por el césped, desasirlos/ de una flor para asirlos a otras flores?// ¿Andar amantes ciegos, olvidados/ de la hora mortal que los circunda,/ soñar que el sueño puede ser el sueño/ sin sobresaltos de una vida nueva?/l ¿ Será un crimen 
Sarà un crimine sedersi la mattina

ad ascoltare la parola delle fontane,

diventare rumore, essere l'eco

di un sussurro senza fine, nascosto?

Un crimine far scivolare sugli alberi

gli occhi, farli scendere dalle coppe,

rovesciarli sull'erba, slegarli

da un fiore per legarli ad altri fiori?

Camminare come amanti ciechi, dimentichi

dell'ora mortale che li circonda,

sognare che il sogno può essere sogno

senza spaventarsi di una nuova vita?

Sarà un crimine pensare che questo sia un crimine,

quando in verità il vero crimine

è quello di non dar respiro al nostro tempo,

commettendo tale crimine quotidianamente?"

L'acqua, elemento vitale per l'uomo, per Alberti è l'essenza imprescindibile che lo ha accompagnato sin dalla nascita e che resulta essere di fondamentale importanza nella sua quotidianità romana, perché innato è lo spirito del "marinero

pensar que esto es un crimen,/cuando en verdad el verdadero crimen/es no darnos respiro nuestro tiempo/para a diario cometer tal crimen?” (¿Será un crimen?, op. cit. pag. 46). Traduzione italiana a cura di Elisabetta Bagli. 
en tierra"31 che mai lo abbandonerà. Questo aspetto permette ad Alberti di poter superare l'afflizione per l'esilio ${ }^{32}$ :

L'acqua delle innumerevoli fontane. Dormo

ascoltando il suo infinito
echeggiare. L'acqua è
qui a Roma il mio sogno.

2

Continua a parlare l'acqua delle fontane

completamente lontana

da tutto, indifferente.

Quel che dice è solo che suona.

3

Acqua di Roma per il mio esilio,

per il mio cuore

tante volte fuori dal suo controllo.

31 "Marinero en tierra" es el título de un libro de poemas de Rafael Alberti pubblicato nel 1924 e che ottenne il Premio nazionale di Poesia nel 1925. Tra i giurati vi era anche Antonio Machado.

32 "Rafael Alberti y la poesía tradicional", Armando López Castro, Universidad de León, pag.110.

33 1- "El agua de las fuentes innumerables. Duermo /oyendo su infinito/resonar. Agua es/aquí en Roma mi sueño.// 2-Sigue charlando el agua de las fuentes/completamente ajena/a todo, indiferente./Lo que dice es tan sólo lo que suena.// 3- Agua de Roma para mi destierro,/para mi corazón/fuera de sus dominios tantas veces.// 4 Agua de Roma para mis insomnios,/esos largos oscuros en que pueblo los techos/de mí, mudas imágenes,/que apenas si conozco./Agua para los pobres, los mendigos, lesos que se abandonan al borde de las fuentes/y se quedan dormidos./Agua para los perros vagabundos,/para todas las bocas sedientas, de pasada,/agua para las flores y los pájaros,/para el cielo volcado con sus nubes,/con su luna, su sol y sus estrellas./Pero por sobre todo,/agua sólo sonido, repetición constante,/agua sueño sin fin,/agua eterna de Roma./Agua". op. cit., pagine. 66-67. Traduzione italiana a cura di Elisabetta Bagli. 
4

Acqua di Roma per le mie insonnie,

quelle lunghe e oscure nelle quali popoli i soffitti

di me, mute immagini,

che appena conosco.

Acqua per i poveri, i mendicanti,

quelli che si abbandonano sul ciglio delle fontane

e lì si mettono a dormire.

Acqua per i cani vagabondi,

per tutte le bocche assetate che passano,

acqua per i fiori e gli uccelli,

per il cielo rovesciato con le sue nubi,

con la sua luna, il suo sole e le sue stelle.

Però e soprattutto

acqua, solo un suono, ripetizione constante,

acqua sogno infinito,

acqua eterna di Roma.

Acqua.

Nel corso della lettura di questa sezione si susseguono molti versi sciolti, scene e canzoni che altro non sono che quadri di una Roma che Alberti ha vissuto dipingendola con la sua particolare sensibilità di artista a $360^{\circ}$. Alberti dice di "non essere arrivato a Roma per sognare", "rompendosi i piedi contro le pietre" 34 , ma

34 "Tú no has llegado a Roma para soñar. Los sueños/ se quedaron tan lejos, que ya ni los divisas,/ ni ellos te buscan ya, pues ya ni te conocen". - "Non sei venuto a Roma per sognare. I tuoi sogni/ sono ormai così indietro che nemmeno li scorgi/ né ti cercano più, perché non ti conoscono". (Tú no has llegado a Roma para soñar, op. cit. pag. 75). Traduzione italiana a cura di Vittorio Bodini. 
di esservi arrivato per cercare quel filo che lo avrebbe riportato a se stesso proprio quando tutto sembrava essere perso, morto o ferito, per essere nel luogo in cui l'acqua e il vento navigano per purificare, per iniziare una nuova vita, dove ancora è tutto buio e vuoto, ma che un giorno sarà luce e pienezza, la sua amata Patria. Per questo si chiede: "Chi si ricorderà di me, quando me ne andrò da Roma?" La sua risposta è quella di domandare a chi lo ha conosciuto. In questa lirica che leggeremo innanzi, possiamo vedere un implicito riferimento al poeta inglese John Keats, che visse e morì a Roma (ora è sepolto presso il cimitero acattolico della città eterna) che volle in epígrafe sulla sua tomba queste parole "Qui giace colui il cui nome venne scritto con l'acqua ${ }^{35 ", ~ m e n t r e ~ p e r ~ A l b e r t i ~ s a r a ̀ ~}$ l'acqua che scorre a scrivere il suo nome sotto un ponte:

"Quando me ne andrò da Roma" 36

Quando me ne andrò da Roma

Chi si ricorderà di me?

Domandino al gatto, domandino al cane e alla scarpa rotta.

Al lampione perduto, al cavallo morto e al balcone ferito.

Al vento che passa, al portone buio

35 "Here lies one whose name was writ in wáter", epitaffio John Keats nel cimitero acattolico di Roma.

36 "Cuando me vaya de Roma/¿Quién se acordará de mí?//Pregunten al gato/pregunten al perro/y al roto zapato.//Al farol perdido,/al caballo muerto/y al balcón herido.//Al viento que pasa,/al portal oscuro/que no tiene casa.//Y al agua corriente/que escribe mi nombre/debajo del puente.//Cuando me vaya de Roma/pregunten a todos por mí." (Cuando me vaya de Roma, op. cit. pag. 76). Traduzione italiana a cura di Elisabetta Bagli. 
che non ha casa.

E all'acqua che scorre

che scrive il mio nome

sotto un ponte.

Quando me ne andrò da Roma

chiedano a tutti di me.

Nel passaggio dal giorno alla notte, si fanno più pressanti i motivi della solitudine e della fugacità della vita. II poeta prende coscienza che, prima o poi, lascerà la città di Roma per ritornare nella propria Patria o per cause naturali. In una delle liriche con lo stesso titolo Nocturno, oltre a porre in evidenza tale sentimenti, Alberti torna anche un po' bambino, scrivendo una canzone la cui cadenza è quella di una ninna nanna o filastrocca. Ne riporto solo un piccolo frammento ${ }^{37}$.

\section{"Notturno"38}

Tieni, tieni la chiave di Roma,

perché in Roma c'è una via,

nella via c'è una casa,

nella casa c'è una stanza,

nella stanza c'è un letto,

nel letto c'è una dama,

una dama innamorata,

\footnotetext{
37 Vid. «Già troppe volte esuli» Letteratura di frontera e di esilio, a cura di Novella di Nunzio e Francesco Ragni Tomo I copyright (C) Università degli Studi di Perugia, AA.VV. Forme di esilio, migrazionem frontera: poesia, Abele Longo Roma, viandanza dell'esilio, Rafael Alberti tradotto da Vittorio Bodini, pag. 116

38 "Toma y toma la llave de Roma,/ porque en Roma hay una calle,/ en la calle hay una casa,/ en la casa hay una alcoba,/ en la alcoba hay una cama,/ en la cama hay una dama,/ una dama enamorada, /que toma la llave, /que deja la cama,/ que deja la alcoba, /que deja la casa,/ que sale a la calle,/ ... (Nocturno, op. cit. pag. 69). Traduzione italiana a cura di Vittorio Bodini.
} 
che prende la chiave,

che lascia il letto,

che lascia la stanza,

che lascia la casa,

che va per la via,

Sempre nel passaggio dalla luce e alla vita quotidiana alla notte, in Rafael Albert si fa più presente la paura della morte, la sente ovunque; per lui la morte è in agguato, dietro ogni angolo, in ogni monumento, in ogni sensazione che prova e vive e "pensa a Keats morto a Roma/ sempre avvolto in un sudario di violette". 39 I sonetti della terza sezione riprendono quel tono umoristico, satirico e caricaturale che abbiamo potuto riscontrare soprattutto nella prima sezione di questa silloge, ma non vi è un dialogo, come accadeva nei sonetti con il Belli, sebbene appaiano le ombre di Quevedo ${ }^{40}$ e di Bertold Brecht ${ }^{41}$ :

"Artrosi (II)"42

Non posso camminare. Sono più zoppo

dello stesso don Francisco de Quevedo.

E il grande dramma romano è che non posso neanche

396 "Pienso en Keats muerto en Roma/Y siempre amortajado entre violetas", op. cit. pag. 48. Traduzione italiana a cura di Vittorio Bodini.

40 Francisco de Quevedo (Madrid, 1580-1645), poeta e scrittore spagnolo considerato forse l'ingegno più vigoroso, inquieto e complesso della letteratura spagnola. Inoltre, è considerato una delle figure più significative del barocco europeo.

41 Bertold Brecht $(1898-1956)$ è stato un drammaturgo, poeta, regista teatrale, naturalizzato austriaco. Considerato uno degli autori più influenti del suo tempo che impersona perfettamente sia dal punto di vista biográfico che artístico. La sua più grande opera fu "Vita di Galileo".

42 "No puedo caminar. Estoy más cojo/que el propio don Francisco de Quevedo./Y el gran drama romano es que ni puedo/Poner ya el pie en el Tiber al remojo." (Artrosis -II, op. cit. pag. 83). Traduzione italiana a cura di Elisabetta Bagli. 
mettere a mollo nel Tevere il mio piede.

"Risposta del tempo"43

\section{A Bertold Brecht}

E nelle notti estive romane,

si sentono ridere dal Vaticano

che affondó, nella notte buia, Galileo.

Fa la sua comparsa anche Don Ramón del Valle Inclán ${ }^{44}$ con tre sonetti intitolati "Notturno (Nocturno)" a lui dedicati. Questi sonetti raggruppati sotto il titolo "(Tre notturni romani con Don Ramón del Valle Inclán (Tres nocturnos romanos con Don Ramón del Valle Inclán)", onorano e ricordano il drammaturgo, scrittore e poeta che visse a Roma per alcuni anni, pemettendoci di vedere la città eterna in un modo ulteriormente differente; Alberti, unendo mondi onirici e fantastici a incubi e tormenti che non si possono interpretare se non attraverso le sue liriche, espressioni di sentimenti autentici e di deliri, ci offre un'ampia e suggestiva visione della realtà popolare romana ${ }^{45}$.

L'ultima sezione chiamata "Poesie con nome (Poemas con nombre)" consiste in otto poesie dedicate a vari artisti visuali della città di Roma, contemporanei e amici di Alberti (Bruno Caruso, Aligi Sassu, Guido Strazza, Carlo Quattrucci, Giuseppe Mazzullo, Corrado Cagli, Umberto Mastroianni, Abel Vallmitjana). Le otto poesie dell'ultima sezione sono scritte dal poeta-pittore, come omaggio alla

\footnotetext{
43 "Y en las romanas noches de verano, /se les siente reír del Vaticano/que hundió en la noche oscura a Galileo." (Respuesta del tiempo- A Bertold Brecht, op. cit. pag. 87). Traduzione italiana a cura di Elisabetta Bagli.

44 Don Ramón del Valle Inclán (1866- 1936) fu un drammaturgo, poeta e romanziere spagnolo che formò parte della corrente letteraria denominata modernismo e, nelle sue ultime opere si può annoverare nella generazione del '98. Viene considerato come uno degli autori chiave della letteratura spagnola del secolo XX.
}

45 “Nocturno 1", “Nocturno intermedio 2", “Nocturno3", op. cit. pagine 84-85-86 
sua vocazione primaria, la pittura, appunto, e come segno di stima nei confronti dei propri amici, senza mai abbandonare sarcasmo e nostalgia, sagacia e inquietudine, risorgendo dalle radici attraverso la parola. In queste liriche, la Roma di Alberti appare un po' sfumata, come se facesse da sfondo a storie che si diluiscono tra schiamazzi e respiri.

Conclusioni

La Roma di Rafael Alberti è complessa e difficilmente si può spiegare se non leggendo i suoi versi. È una Roma che attraversa il tempo e lo spazio, che abbraccia le tematiche più disparate, utilizzando il verseggiare anche per esprimere idee politiche da denunciare o per far conoscere la sua idea sulla religione e la Chiesa, vagando dalle barzellette agli aneddoti, dal racconto dialogato alle storie popolari, entrando nel suo intimo, dipinto da amara melanconia per l'esilio.

Nelle liriche contenute in questa silloge si avverte Roma come una città in embollizione, in continua frenesia, che acceca il viandante al solo passaggio per le strade, una città dalla storia millenaria che è oltraggiata quotidianamente dai rumori e dalle situazioni che nulla hanno a che vedere con i suoi monumenti e proprio per questo resulta essere ancora più attraente e autentica. Alberti ci parla di colombe e di gatti "sacri" che giocano e vengono sfamati dalla "gattara" di turno, di lucertole che dormono stese sulle antiche pietre a prendere il sole, del mascherone che adorna una fontana, di San Pietro pescatore, di un cocchiere con il suo cavallo...

Il paesaggio urbano di Alberti è molto articolato: i personaggi- reali o immaginari - o quelli mitologici escono fuori dai libri per mostrarsi in tutta la loro "umanità" (Leda, Polifemo, Giordano Bruno, la Lozana andaluza...)

In "Roma, pericolo per i viandanti", Rafael Alberti ci regala la sua particolare visione di Roma dalla quale emergono gli aspetti ludici e umoristici, nostalgici e umani che caratterizzano il suo essere artista e tutta la sua produzione letteraria e pittorica. 


\section{Bibliografia:}

- $\quad$ "Roma, peligro para caminantes", Rafael Alberti, Ed. Seix Barral, ed. 1968, primera edición en la Biblioteca Breve de Bolsillo: abril de 1976. 1aㅡ reimpresión: mayo de 1977.

- II Congreso Internacional de Literatura y Cultura Españolas Contemporáneas "Roma, peligro para caminantes. La representación de una ciudad en estratos" - Ester Hernández Palacios Mirón Universidad Veracruzana.

"Rafael Alberti, caminante en Roma", Marta Villarino, pag 258/ CELEHIS

"Rafael Alberti y la poesía tradicional", Armando López Castro, Universidad de León, pag.110.

- «Già troppe volte esuli» Letteratura di frontera e di esilio, a cura di Novella di Nunzio e Francesco Ragni Tomo I copyright @ Università degli Studi di Perugia, AA.VV.

Forme di Esilio, migrazione, frontera: Poesia, Abele Longo

Roma, viandanza dell'esilio, Rafael Alberti tradotto da Vittorio Bodini.

Alberti en Roma - Guia de Italia (El País Semanal, 21 de Julio de 2002). Terenci Moix.

EI País- TRIBUNA: "La arboleda perdida" - De Buenos Aires al Trastevere 11 de noviembre de 1984, autor Rafael Alberti.

Homenaje a Alberti y representación en Cádiz de "La lozana andaluza", EI País, 2 de agosto de 1980 El poeta adaptó para el teatro la obra de Francisco Delicado. 